\title{
Análisis del impacto de becas otorgadas por la Sociedad Argentina de Pediatría en los últimos 20 años (1995-2015)
}

\author{
Assessment of the impact of grants awarded by the Sociedad \\ Argentina de Pediatria during the last 20 years (1995-2015)
}

Dra. Paula Domínguez ${ }^{a}$ Dra. Vanesa Castellano ${ }^{a}$ María B. Rey y Dra. Silvia Caino ${ }^{a}$

\begin{abstract}
RESUMEN
LaSociedad Argentina de Pediatría otorga becas a pediatrasjóvenes con elobjetivo de perfeccionar el desempeño e incentivar la investigación.

Objetivos. Describir las características de las becas otorgadas y evaluar la proporción de proyectos publicados y de becarios de perfeccionamiento que permanecieron en áreas relacionadas con su beca.

Material y método. Estudio descriptivo. La Subcomisión de Becas y Premios elaboró una encuesta, que fue enviada por correo electrónico a los becarios en forma individual.

Resultados. Se otorgaron 59 becas de investigación (1995-2015). Respondieron la encuesta 47 becarios. Alcanzaron la publicación 14 proyectos. Haber realizado la beca en un hospital pediátrico se asoció a publicación odds ratio $13,8(1,6-118), \mathrm{p}=0,01$. Se otorgaron 132 becas deperfeccionamiento (2005-2015). Respondieron la encuesta 84 becarios. El $85 \%$ continuaba trabajando en la misma área de su beca.

Palabras clave: publicaciones, informe de investigación, becas.
\end{abstract}
ABSTRACT
The ArgentineSociety of Pediatrics awards grants to young pediatricians, aimed at improving performance and encouraging research.
Purpose. To describe the details of grants awarded; to analyze the proportion of projects that were published and of grantees that remained in areas related to their grant.
Material and method. Descriptive study, through a self-administered survey.
Results. 59 research grants were awarded (1995-
2015). The survey was answered by 47 grantees; 14 projects reached publication. Having completed the research at a Pediatric Hospital was associated with publication odds ratio 13,8 (1,6-118), $\mathrm{p}=0,01 ; 132$ educational improvement grants were awarded (2005-2015). The survey was answered by 84 grantees. The $85 \%$ continue working in the same area of their grant.
Key words: publications, research report, fellowships and scholarships.
http: / / dx.doi.org/10.5546/ aap.2019.S255
Cómo citar: Domínguez P, Castellano V, Rey MB Caíno S. Análisis del impacto de becas otorgadas por la Sociedad Argentina de Pediatría en los últimos 20 años (1995-2015). Arch Argent Pediatr 2019;117 Supl 6: S255-S263.

a. Subcomisión de

Becas y Premios,

Sociedad Argentina de Pediatría.

Correspondencia:

Dra. Paula Domínguez: pau.dom@gmail.com

Financiamiento:

Ninguno.

Conflicto de intereses: Ninguno que declarar.

Recibido: 12-3-2019

Aceptado: 7-6-2019

\section{INTRODUCCIÓN}

Desde hace más de 20 años, la Sociedad Argentina de Pediatría (SAP) otorga becas de perfeccionamiento e investigación destinadas a pediatras jóvenes de todo el país con el objetivo de perfeccionar el desempeño e incentivar la investigación. El número de aspirantes y las becas otorgadas se modificó a través de los años, y se otorgan, actualmente, 8 becas anuales de investigación y 6 becas semestrales de perfeccionamiento.

La selección de los becarios está coordinada por la Subcomisión de Becas y Premios (SByP), y realizada a través de un jurado conformado por miembros de dicha Subcomisión, Subcomisión de Investigación, Educación Continua de entidad matriz y de filiales, Fundación SAP y comités nacionales. ${ }^{1}$

Desde sus orígenes, las becas financiadas por la SAP fueron publicadas en los libros de actas y memorias, pero no se ha encontrado una verdadera evaluación de su impacto. La evaluación de cualquier programa debe producirse en torno al análisis de sus resultados, entendidos estos como efectos reales sobre sus destinatarios. En el caso que nos ocupa, serían la publicación del trabajo realizado por el becario durante la beca de investigación y la permanencia en la especialidad para los becarios de perfeccionamiento.

Evaluar la tendencia a lo largo de un período suficientemente extenso sería de utilidad para generar estrategias destinadas a mejorar este programa y analizar las tendencias históricas. 


\section{OBJETIVOS}

- Describir las características de las becas otorgadas por la SAP de 1995 a 2015.

- Evaluar la proporción de proyectos becados que alcanzaron la publicación como texto completo y los factores involucrados con la no publicación.

- Evaluar la proporción de becarios de perfeccionamiento que permanecieron en áreas relacionadas con su beca.

\section{MATERIAL Y MÉTODOS}

Se realizó un estudio descriptivo y analítico que incluyó todas las becas de investigación desde 1995 a 2015 y las de perfeccionamiento desde 2005 a 2015, otorgadas por la SAP.

Se identificó a los becarios a través de los libros de actas de la SByP, y de las memorias de la SAP. De los registros de la SAP, se obtuvo el año de otorgamiento de la beca, la filial de procedencia del becario, el lugar de realización y el tema de la beca. La SByP elaboró una encuesta (Anexos 1 y 2), la cual fue enviada por correo electrónico a cada uno de los becarios en forma individual.

Arbitrariamente, se seleccionaron las siguientes medidas de impacto: 1 . para las becas de investigación, la publicación del trabajo realizado por el becario; 2 . para las becas de perfeccionamiento, la permanencia en el tema o especialidad de la beca. Se consideró publicado un trabajo de investigación cuando fue efectuado en una publicación científica periódica reconocida con ISSN.

Los aspectos indagados en la encuesta fueron la difusión de los proyectos de investigación (presentación en congresos, publicación), las causas de no publicación, la permanencia en la especialidad, el regreso al lugar de residencia, la percepción acerca de la utilidad de la beca y las características de los becarios (residencia básica, formación posbásica, publicaciones previas).

Análisis estadístico: Se realizó el análisis descriptivo, que expresó las variables categóricas como números absolutos. Se calcularon las tasas de becas otorgadas anuales (número de becas / 1000 afiliados / año) y por región. Se utilizó la prueba de chi $^{2}$ para evaluar la asociación entre la característica de la beca (lugar de realización, procedencia del becario) y la publicación o permanencia en la especialidad, según correspondiera. Se calcularon los odds ratio $(O R)$ con sus intervalos de confianza (IC) del $95 \%$, nivel de significación de p menor de 0,05. Se utilizó el programa IBM SPSS 21.
Consideraciones éticas: Se cumplió con la Ley de Protección de Datos Personales ( ${ }^{\circ}$ 25326), lo que garantizó una adecuada disociación de datos filiatorios. En la encuesta, se informó sobre los alcances de la investigación, considerando su participación como aceptación. Se obtuvo la aprobación de la Comisión Directiva de la SAP.

\section{RESULTADOS}

\section{Becas de investigación}

Se otorgaron 59 becas de investigación en el período 1995-2015. Fueron solicitadas desde la región Metropolitana en su mayoría $(n=48)$, seguido de la región Pampeana Norte $(n=3)$ y Centro-Cuyo ( $\mathrm{n}=2)$ (Figura 1).

La tasa global (becas totales otorgadas / 1000 afiliados de la SAP / año) fue de 0,27. Al considerar las tasas promedio por región, se observó que las de las regiones Metropolitana y Patagónica Atlántica presentaron más altas que la global (0,53 y 0,54, respectivamente). La región Pampeana, 0,16, y las regiones de Cuyo, Litoral y Noroeste Argentino (NOA) presentaron las tasas más bajas en comparación con la global $(0,06,0,04$ y 0,04, respectivamente).

Los temas de investigación más frecuentes fueron referentes a infectología, neumonología, neonatología, adolescencia y nefrología. Las instituciones donde más frecuentemente se realizaron las becas fueron el Hospital General de Niños Pedro de Elizalde $(n=13)$, el Hospital General de Niños Ricardo Gutiérrez $(\mathrm{n}=12)$, el Hospital de Pediatría Garrahan $(\mathrm{n}=10)$, el Hospital Italiano $(n=4)$, el Hospital Nacional Prof. A. Posadas $(n=3)$ y el Hospital Sor María Ludovica $(\mathrm{n}=2)$.

Respondieron la encuesta 47 becarios (el $80 \%$ ). De ellos, 33 (el 70,2\%) continuaban, hasta el momento de la encuesta, trabajando en la misma institución en la cual habían realizado la beca; 35 (el 74,5\%) se desempeñaban en la misma área de su beca y 37 becarios finalizaron la investigación en el tiempo estipulado (becas de 1 año). La causa principal de no finalización fue "problemas para alcanzar el tamaño muestral".

Alcanzaron la publicación completa 14 proyectos becados (el $29 \%$; IC $95 \%$ : 18,643,9). Siete de ellos fueron publicados en Archivos Argentinos de Pediatría. Otras 2 publicaciones se realizaron en revistas indizadas en Pubmed (Pediatric Pulmonology y Thorax) y el resto, en publicaciones locales (Revista de Salud Pública, revista Medicina Infantil del Hospital Garrahan, 
Revista Pediátrica Elizalde y libro UNICEF/ Ministerio de Salud).

Las causas más frecuentes para la no publicación fueron la falta de tiempo $(\mathrm{n}=18)$ y el tamaño muestral insuficiente $(\mathrm{n}=7) .37$ trabajos fueron presentados en algún evento científico; la mayoría $(\mathrm{n}=26)$, a nivel nacional.

Respecto al perfil de los becarios, 30 realizaron su residencia básica en un hospital pediátrico (15 en el Elizalde, 12 en el Gutiérrez, 3 en el Garrahan); 28 realizaron la residencia posbásica; 7, un curso superior de especialista; 10 , otras becas y concurrencia. Solo 2 no tenían formación posbásica. 18 refirieron publicaciones previas al momento del otorgamiento de la beca de investigación.

Con respecto a la percepción de los becarios acerca de la utilidad de la beca recibida,
23 consideraron que había impactado "mucho" o "en gran medida" en su desarrollo profesional; 16, que el impacto había sido "suficiente", y 8, "nada" o "moderadamente".

Al analizar los probables factores asociados a la publicación, haber realizado la beca de investigación en los hospitales pediátricos Elizalde, Gutiérrez o Garrahan (E/G/G) se asoció a una mayor probabilidad de publicación, con $O R$ $13,8(1,6-118), \mathrm{p}=0,01$.

No se encontró asociación entre la publicación y la región desde la cual se había solicitado la beca, seguir trabajando en la misma institución o el lugar de la residencia básica (Tabla 1).

\section{Becas de perfeccionamiento}

Se otorgaron 132 becas de perfeccionamiento en el período 2005-2015 (Figura 2). Las regiones

FIgura 1. Distribución de las becas de investigación según la región

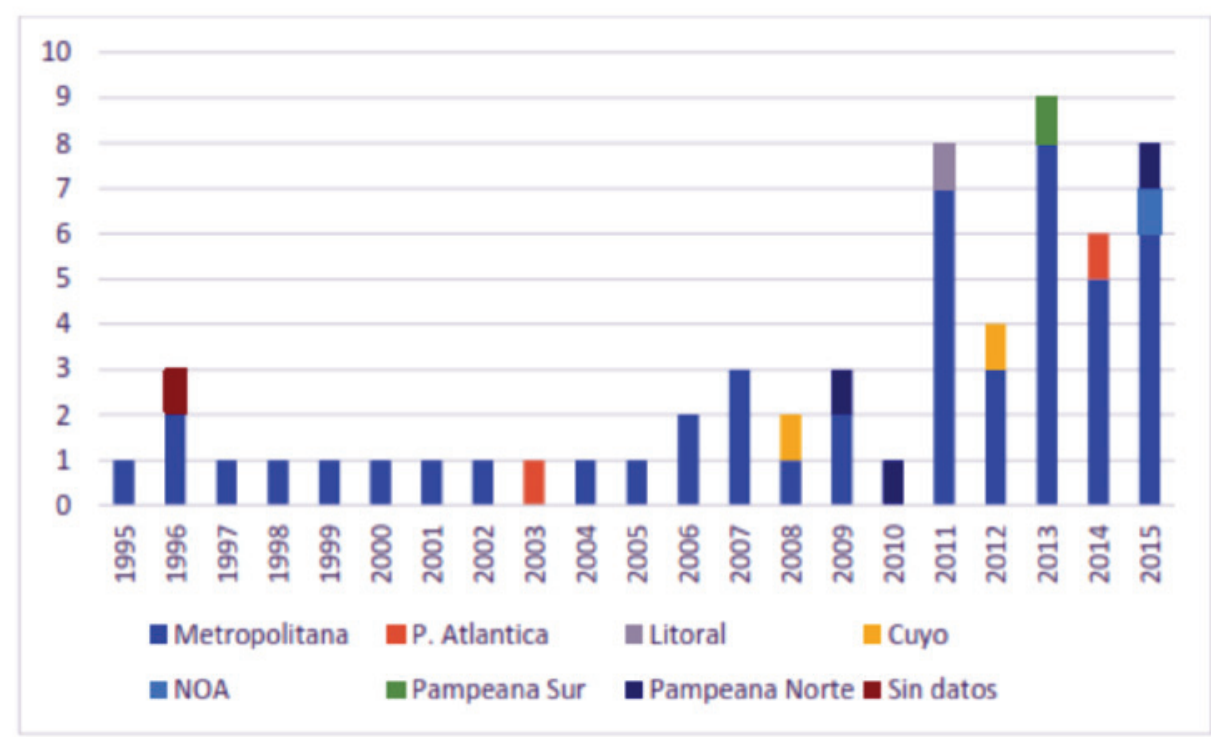

NOA: Noroeste Argentino.

TABla 1. Asociación entre las distintas variables y la publicación de las becas de investigación

\begin{tabular}{lcccc}
\hline & Publicado & No publicado & OR & $p$ \\
\hline Región Metropolitana & 13 & 27 & $2,8(0,3-26,5)$ & 0,6 \\
Otras regiones & 1 & 6 & $13,8(1,6-118)$ & 0,01 \\
Beca E/G/G* & 13 & 16 & & \\
Beca de otra institución & 1 & 17 & $0,67(0,1-2,5)$ & 0,8 \\
Continúa en la misma institución & 9 & 24 & $1,9(0,3-10,4)$ & 0,7 \\
No continúa en la misma institución & 5 & 9 & 25 & 0,3 \\
Finalizó la beca a tiempo & 12 & 8 & $2,7(0,6-11,5)$ & \\
No finalizó la beca a tiempo & 2 & 19 & & \\
Residencia básica en E/G/G* & 11 & 14 & & \\
Residencia básica en otra institución & 3 & &
\end{tabular}

* E/G/G: Hospital General de Niños Pedro de Elizalde, Hospital General de Niños Ricardo Gutiérrez y Hospital de Pediatría Garrahan. 
desde las cuales fueron solicitadas se presentan en la Tabla 2.

La tasa global (becas totales otorgadas / 1000 afiliados de la SAP/año) fue de 0,83. Las tasas de las regiones Patagónica Andina $(1,21)$, Metropolitana $(1,03)$ y NOA $(0,88)$ fueron superiores a la media global. Las regiones Patagónica Atlántica $(0,66)$, Cuyo $(0,58)$, Litoral $(0,44)$, Noreste Argentino (NEA) $(0,42)$ y Pampeana $(0,21)$ presentaron tasas inferiores a la global.

Las instituciones más frecuentemente elegidas para realizar la beca fueron el Hospital General de Niños Ricardo Gutiérrez $(\mathrm{n}=25)$, el Hospital Nacional Prof. A. Posadas ( $n=23)$, el Hospital de Pediatría Garrahan $(\mathrm{n}=21)$, el Hospital General de Niños Pedro de Elizalde $(\mathrm{n}=11)$, el Hospital Italiano de Buenos Aires $(n=10)$, el Hospital General de Agudos Dr. C. Argerich $(n=6)$, el Hospital Pediátrico Dr. Humberto Notti $(n=5)$.

Las áreas de capacitación más frecuentes fueron Nutrición, Cardiología, Adolescencia, Gastroenterología/Hepatología, Crecimiento y Desarrollo, y Cuidados Paliativos.

Respondieron la encuesta 84 becarios (el $64 \%$ ). De ellos, 67 residían en la misma región en la cual habían solicitado realizar la beca. De las 24 becas solicitadas desde las filiales, 13 regresaron a su lugar de residencia luego de haber finalizado el período de beca, 7 fueron realizadas en instituciones de la misma filial donde residía el becario y 4 no regresaron a su lugar de residencia luego de haber finalizada la beca en el área Metropolitana. 71 becarios continuaban trabajando en la misma área de su beca.

En cuanto al perfil de los becarios, 36 realizaron su residencia básica en un hospital pediátrico o institución privada de la Ciudad Autónoma de Buenos Aires (CABA) (33 y 3, respectivamente) y 46 , en hospitales fuera de la CABA (27 instituciones de la provincia de Buenos Aires y 19 en otras provincias). Con respecto a la formación posbásica, 31 becarios realizaron un curso superior; 26 , residencia, y 10, otra beca,

TABLa 2. Distribución de las becas de perfeccionamiento según la región de solicitud $(n=132)$

\begin{tabular}{lc}
\hline Región & Nro. de becas \\
\hline Metropolitana & 75 \\
Centro-Cuyo & 13 \\
NOA & 13 \\
Litoral & 7 \\
Patagónica Andina & 5 \\
NEA & 3 \\
Pampeana Norte & 2 \\
Pampeana Sur & 2 \\
Patagónica Atlántica & 2 \\
Sin datos & 10 \\
\hline
\end{tabular}

NOA: Noroeste Argentino; NEA: Noreste Argentino.

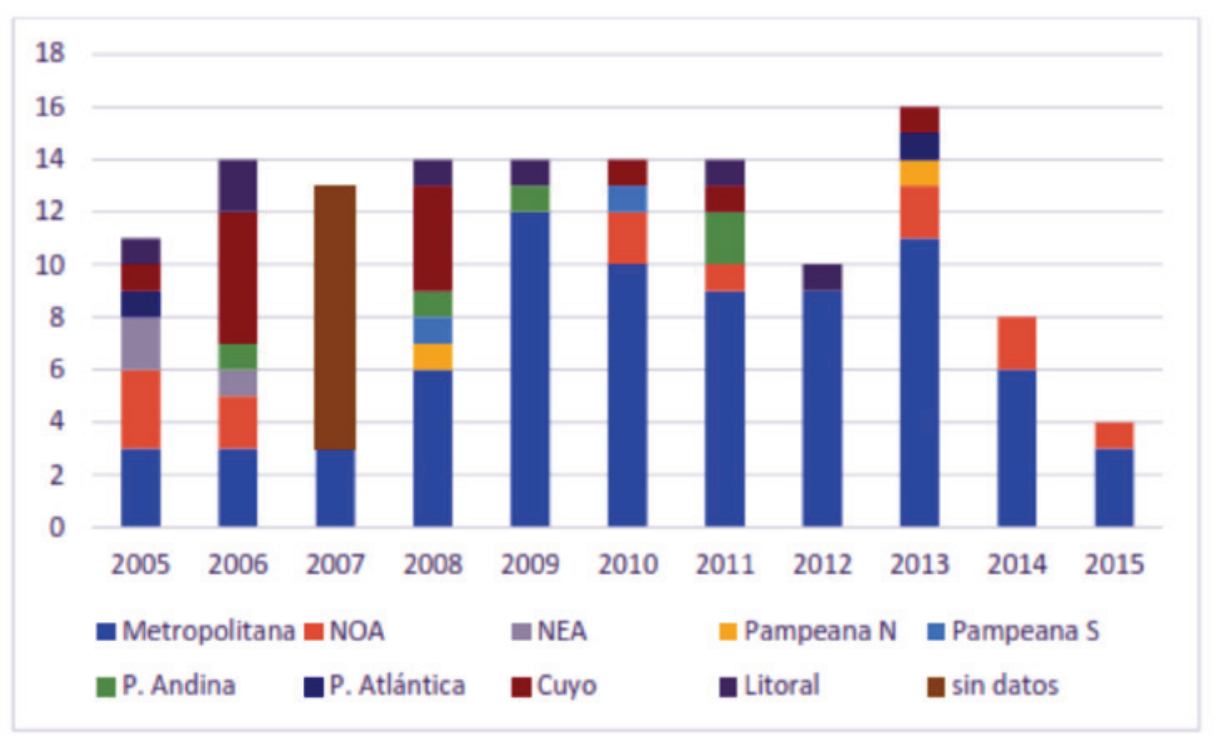

NOA: Noroeste Argentino; NEA: Noreste Argentino. 
concurrencia o maestrías. 15 becarios no refirieron formación posbásica.

Respecto a la utilidad percibida por los becarios, 65 consideraron que las competencias adquiridas durante la beca de perfeccionamiento fueron útiles "en gran medida" para mejorar la atención en su ámbito laboral. 71 becarios respondieron que la beca había tenido un impacto positivo en su desarrollo profesional y 13 consideraron que no había tenido impacto o que había sido moderado.

\section{DISCUSIÓN}

Este trabajo permitió realizar un análisis descriptivo y una evaluación del impacto de las becas otorgadas por la SAP, por lo que fue una herramienta de interés para visibilizar la tendencia de las becas otorgadas de los últimos años, analizar en forma objetiva el impacto del programa de becas y la percepción de utilidad de los becarios para su desarrollo profesional.

De los resultados sobre el número de becas otorgadas surge que su distribución respecto a las filiales de la Sociedad es heterogénea. El cálculo de la tasa global y por filial permitió ajustar por región y por número de afiliados, lo que demostró que las becas se habían otorgado, principalmente, a becarios de las regiones Metropolitana, Patagónica Atlántica y NOA. Sin embargo, y debido a cuestiones vinculadas con el registro, solo se pudieron analizar los proyectos que adjudicaron la beca de la SAP y quedaron fuera de este análisis aquellas solicitudes que no alcanzaron los estándares necesarios para el otorgamiento de la beca. Esto podría significar un sesgo, ya que no se puede establecer si las regiones con menor tasa de otorgamiento de becas también fueron las que menos las solicitaron.

En los últimos 10 años, aumentó progresivamente el número de becas de investigación otorgadas, mientras que el número de becas de perfeccionamiento se mantuvo estable. Esto podría estar relacionado con la necesidad creciente de generar evidencia científica y con el fortalecimiento de la investigación en pediatría, en concordancia con el apoyo de la SAP en este campo.

En relación con los temas de investigación de las becas, fueron, en su mayoría, referidos a especialidades pediátricas. Sería necesario establecer estrategias destinadas a promover la investigación en áreas de pediatría general o atención primaria de la salud.

La publicación de un trabajo científico es el objetivo final de toda investigación. Sin embargo, es sabido que la mayoría de las investigaciones no lo alcanzan. En este trabajo, surge que el $30 \%$ de las becas de investigación alcanzaron la publicación. Este dato es inferior al referido para las publicaciones de los trabajos que se presentan en los congresos de especialidades médicas $(\mathrm{el} 44 \%)^{2,3}$

La causa mencionada como limitante para la publicación fue la falta de tiempo, en coincidencia con otros estudios. ${ }^{4} \mathrm{~A}$ pesar de que el programa de becas de la SAP incluye tiempo protegido para la investigación, esto continúa siendo una barrera. Sería necesario realizar un análisis más exhaustivo, a priori, acerca de la factibilidad de la realización de los proyectos aspirantes durante el tiempo de beca, así como también destinar tiempo para alcanzar la publicación dentro del cronograma de cada proyecto.

Al respecto, Rothberg y col., muestran que la implementación de un programa de investigación, estructurado en distintas etapas, durante la residencia, logró incrementar tanto la presentación como la publicación de las investigaciones realizadas por residentes. ${ }^{5} \mathrm{La}$ $\mathrm{SAP}$, a través de su programa de becas, pretende, del mismo modo, incentivar la formación y la investigación, ofreciendo un espacio financiado, con tiempo protegido y supervisado para el desarrollo de estas actividades.

Con respecto a las becas de perfeccionamiento, el otorgar espacios de formación posterior a la formación en Pediatría, principalmente, permite ampliar las competencias en un área determinada, fomentar el desarrollo de profesionales de acuerdo con las prioridades en salud y transferir las competencias alcanzadas a su lugar de trabajo.

Sin embargo, de este análisis surge que la mayoría de estas becas son solicitadas y realizadas en la región Metropolitana. De este modo, se cumple parcialmente con el objetivo de formar profesionales que puedan desempeñarse en otras regiones. En este marco, se plantea la necesidad de fomentar la participación de aspirantes desde las filiales.

Sería de utilidad, en futuras encuestas, profundizar acerca de los nuevos conceptos o herramientas adquiridas por los becarios de perfeccionamiento y contar, además, con información de los directores de beca, a fin de mejorar la factibilidad de los proyectos.

\section{CONCLUSIÓN}

La SAP otorgó 59 becas de investigación y 132 
de perfeccionamiento en el período estudiado. Alcanzaron la publicación completa el $30 \%$ de los proyectos de investigación. El $85 \%$ de los becarios de perfeccionamiento continúa trabajando en la especialidad de la beca.

\section{REFERENCIAS}

1. Sociedad Argentina de Pediatría. Reglamento de Becas. [Acceso: 10 de junio de 2019]. Disponible en: www.sap. org.ar/uploads / archivos / general / files_reglamento-becas-19_1546468244.pdf.

2. Scherer RW, Langenberg P, Von Elm E. Full publication of results initially presented in abstracts. Cochrane Database Syst Rev. 2007; (2):MR0000005.

3. Domínguez P, Ossorio MF, Cuestas E, Giglio N, et al. Publicación de trabajos presentados en los Encuentros Nacionales de Investigación Pediátrica de la Sociedad Argentina de Pediatría: factores involucrados. Arch Argent Pediatr. 2016; 114(4):351-4.

4. Song F, Loke Y, Hooper L. Why are medical and healthrelated studies not being published? A systematic review of reasons given by investigators. PLoS One. 2014; 9(10):e110418.

5. Rothberg MB, Kleppel R, Friderici JL, Hinchey K. Implementing a resident research program to overcome barriers to resident research. Acad Med. 2014; 89(8):1133-9. 


\section{ANEXO 1. \\ Encuesta para becarios de investigación}

1. ¿Actualmente, trabaja en la misma institución donde realizó la beca de investigación?

Sí. O No. O

Especifique la institución en la cual realizó la beca:

2. ¿Actualmente, trabaja en el área en que desarrolló la beca de investigación?

Sí. O No. O

Especifique el tema o área de su beca:

3. ¿Durante el período de beca, terminó el proyecto de investigación?

Sí. O No. O

Si su respuesta fue negativa, indique cuál/es cree que fue/ron la/s causa/s:.

4. ¿Su trabajo fue publicado en alguna revista científica?

Sí. O No. O

Si su respuesta fue afirmativa, indique el nombre del artículo, revista, volumen, páginas, año:

5. Si su respuesta fue negativa, indique cuál considera que fue la razón más importante que impidió la publicación de su investigación (marque solo una opción):

Falta de interés.

Falta de tiempo.

O

O

O

O

O

O

O

O

Dificultad con el análisis estadístico.

Pesimismo de los autores con respecto a la aceptación para su publicación. $\bigcirc$

Dificultad en el financiamiento.

No aceptado para su publicación (rechazado).

Otra causa (especifique):

6. ¿Su trabajo fue presentado en algún congreso o reunión científica?

Sí (especifique el evento):

No. O

7. ¿Dónde realizó su residencia básica?

8. ¿Realizó alguna formación posbásica?

Residencia. $\bigcirc$

Curso superior. $O$

Otra (especifique):

No. $O$

9. ¿Publicó otros trabajos de investigación previos a la beca otorgada?

Sí. O

No. $O$

Si su respuesta fue positiva, por favor, indique el nombre del artículo, revista, volumen, páginas, año (de, al menos, dos de los trabajos publicados):

10. ¿En qué medida siente que la beca impactó en su desarrollo profesional?

Nada.

Moderadamente. $\bigcirc$

Lo suficiente. $\mathrm{O}$

Mucho. O

En gran medida. 


\section{ANEXO 2}

Encuesta para becarios de perfeccionamiento

1. ¿Desde qué región de la SAP solicitó su beca de perfeccionamiento?

Metropolitana.

Noreste.

Noroeste.

Pampeana Norte.

Pampeana Sur.

Patagónica Andina.

Patagónica Atlántica.

Centro-Cuyo.

Litoral.

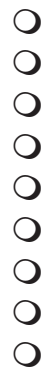

2. ¿En qué institución realizó la beca de perfeccionamiento?

3. En el caso de no haber realizado la beca en la misma región, ¿regresó luego a aplicar las competencias adquiridas a su lugar de residencia?

Sí.

No (especifique la causa):

4. ¿Continuó trabajando en el área relacionada con la beca de perfeccionamiento otorgada?

Sí, hasta la actualidad.

Sí, por un tiempo (menos de 2 años).

No.

Especifique el área o tema de la beca otorg ada:

5. Si su respuesta fue negativa, indique cuál considera que fue la razón más importante que le impidió seguir desarrollándose en el área (marque solo una opción):

Falta de interés personal.

Falta de interés institucional.

Recurso no necesario en el lugar de trabajo.

Falta de tiempo.

Otro (especifique):

6. ¿En qué lugar desempeña actualmente su actividad asistencial? Indique la opción en la cual trabaja la mayor cantidad de su tiempo:

En la misma institución donde realizó la beca.

En otra institución, sector público, área Metropolitana.

En otra institución, sector privado, área Metropolitana.

En otra institución, sector público, filial del interior del país.

En otra institución, sector privado, filial del interior del país.

No trabaja en el área de asistencia médica.

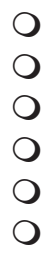

7. Las competencias adquiridas durante la beca de perfeccionamiento, ¿en qué medida le fueron de utilidad para mejorar la atención en su ámbito laboral? Marque la opción que más represente:

En gran medida.

Moderadamente.

No fueron útiles.

8. ¿En qué institución realizó su residencia básica? 
9. ¿Realizó alguna formación posbásica luego de la beca? Indique la especialidad e institución: Residencia.

Curso superior.

O

No.

○

Otro:

10. ¿En qué medida siente que la beca impactó en su desarrollo profesional?

Nada.

Moderadamente.

Lo suficiente.

0

Mucho.

O

En gran medida.

$\bigcirc$

O 